\title{
Erratum zu: Die Repräsentation der Bürgerinnen und Bürger durch organisierte Interessen in Deutschland
}

\author{
D. Pakull $\cdot$ F. Goldberg $\cdot$ P. Bernhagen
}

Online publiziert: 9. Juli 2020

(C) Deutsche Vereinigung für Politikwissenschaft 2020

\section{Erratum zu:}

Politische Vierteljahresschrift 2020

https://doi.org/10.1007/s11615-020-00253-3

Dieser Beitrag wurde versehentlich mit einer veralteten Version von Abb. 4 online publiziert. Der Beitrag wurde aktualisiert.

Wir bitten dieses Versehen zu entschuldigen.

Die Online-Version des Originalartikels ist unter https://doi.org/10.1007/s11615-020-00253-3 zu finden.

D. Pakull $(\bowtie) \cdot$ F. Goldberg $\cdot$ P. Bernhagen

Institut für Sozialwissenschaften, Universität Stuttgart, Breitscheidstr. 2, 70174 Stuttgart,

Deutschland

E-Mail: dominic.pakull@sowi.uni-stuttgart.de 